\title{
Bounds on CP-violating Higgs-gluon interactions: the case of vanishing light-quark Yukawa couplings
}

\section{Ulrich Haisch and Amando Hala}

Max Planck Institute for Physics, Föhringer Ring 6, München 80805, Germany

E-mail: haisch@mpp.mpg.de, ahala@mpp.mpg.de

ABSTRACT: We investigate CP-violating interactions involving the Higgs boson and gluons within an effective field theory approach, focusing on the specific class of new-physics scenarios where the Yukawa couplings of light quarks are zero or strongly suppressed compared to the standard-model expectations. We compute the contributions of the most relevant higher-dimensional operators of Weinberg type to the electric dipole moment of the neutron (nEDM), which are induced by Feynman diagrams that involve an effective CP-violating Higgs-gluon coupling and top-quark loops. The resulting nEDM sensitivities and prospects are discussed and compared to the existing and expected LHC bounds. We find that future $\mathrm{nEDM}$ searches can set non-trivial constraints on CP-violating Higgs-gluon interactions even if the Higgs only couples to the third generation of quarks.

KeYwords: Beyond Standard Model, CP violation, Perturbative QCD

ArXiv EPrint: 1909.09373 


\section{Contents}

1 Motivation 1

2 Calculation 3

2.1 Dimension-six contribution 4

2.2 Dimension-eight contribution 6

$\begin{array}{llr}3 & \text { Discussion } & 7\end{array}$

$\begin{array}{ll}\text { A Case of universal theories } & 9\end{array}$

\section{Motivation}

It is known since more than a decade $[1,2]$ that measurements of the kinematic properties of the Higgs boson and the associated jet spectra provide experimental probes of the $\mathrm{CP}$ structure of the couplings between the Higgs and gauge bosons. In the context of the standard model effective field theory (SMEFT) [3, 4] one CP-violating dimension-six operator, that has been constrained using LHC data [5-8], is

$$
\mathcal{L}_{\phi \tilde{G}}=-g_{s}^{2} \phi^{\dagger} \phi \tilde{G}_{\mu \nu}^{a} G^{a \mu \nu} C_{\phi \tilde{G}} .
$$

Here $g_{s}$ denotes the strong coupling constant, $\phi$ is the standard model (SM) Higgs doublet, $G_{\mu \nu}^{a}$ is the QCD field strength tensor and $\tilde{G}^{a \mu \nu}=1 / 2 \epsilon^{\mu \nu \rho \lambda} G_{\rho \lambda}^{a}$ with $\epsilon^{0123}=+1$ is its dual. Notice finally that the Wilson coefficient $C_{\phi \tilde{G}}$ introduced in (1.1) carries mass dimension -2 , meaning that it can be measured in $1 / \mathrm{GeV}^{2}$.

Since searches for electric dipole moments (EDMs) are also known to place stringent constraints on any new-physics scenario with additional sources of CP violation (see [924] for reviews and recent discussions) the low-energy constraints on effective operators of the form (1.1) have also been considered [25-31]. In fact, the recent article [31] performed a comprehensive study of the relative strengths and complementarity of collider and low-energy measurements in probing CP-violation in Higgs-gauge boson interactions. Employing a SMEFT description and working in the context of so-called universal theories [32-34], i.e. theories in which mainly the couplings between the SM Higgs and gauge bosons are modified by new dynamics, it was found in the latter work that in a singleoperator analysis the existing EDM limits leave very little room for observing CP violation in the Higgs sector at the LHC. Including all relevant dimension-six CP-violating operators, it was furthermore established that the EDM searches enforce strong correlations among Higgs-gauge boson couplings, which barring intricate cancellations lead again to stringent bounds on the individual Wilson coefficients. Similar conclusions where drawn in [30] where only the limits arising from the electron EDM (eEDM) have been studied. 
In this article we would like to point out a simple way of how-to relax the constraints obtained in $[30,31]$. In contrast to the latter articles, we will not assume that the newphysics modifications are confined to the Higgs-gauge boson sector, but also allow for effects in the Yukawa sector. Specifically, we will consider the following dimension-six SMEFT terms

$$
\mathcal{L}_{\phi q}=-Y_{d} \phi^{\dagger} \phi \bar{Q}_{L} \phi d_{R} C_{\phi d}-Y_{u} \phi^{\dagger} \phi \bar{Q}_{L} \tilde{\phi} u_{R} C_{\phi u}+\text { h.c. }
$$

where we have employed the shorthand notation $\tilde{\phi}^{i}=\epsilon_{i j}\left(\phi^{j}\right)^{*}$ with $\epsilon_{i j}$ totally antisymmetric and $\epsilon_{12}=+1$. The Yukawa couplings $Y_{d}$ and $Y_{u}$ are matrices in flavour space and a sum over flavours indices is implicit in (1.2). Finally, $Q_{L}$ denote left-handed quark doublets, while $d_{R}$ and $u_{R}$ are right-handed fermion singlets of down-quark and up-quark type, respectively.

After electroweak symmetry breaking (EWSB) the dimension-six operators in (1.2) modify the couplings of the Higgs boson to quarks. Assuming that new-physics is minimally flavour violating [35] and that the Wilson coefficients $C_{\phi u}$ and $C_{\phi d}$ are real, ${ }^{1}$ each SM quark Yukawa coupling gets rescaled by an independent factor

$$
\kappa_{q} \simeq 1+v^{2} C_{\phi q},
$$

where $q=t, b, c, s, d, u$ and $v \simeq 246 \mathrm{GeV}$ denotes the electroweak (EW) vacuum expectation value. The coupling modifiers $\kappa_{q}$ or equivalently the Wilson coefficients $C_{\phi q}$ can be constrained by LHC Higgs physics. In the case of the top and bottom quark, our knowledge of Yukawa interactions has undergone a revolution in the last year, since the ATLAS and CMS collaborations have independently observed $p p \rightarrow t \bar{t} h$ production $[36,37]$ and the $h \rightarrow b \bar{b}$ decay $[38,39]$. Combining direct and indirect information on the Higgs properties into a global fit ATLAS [40] finds the following $68 \%$ confidence level (CL) limits ${ }^{2}$

$$
\kappa_{t}=1.02_{-0.10}^{+0.11}, \quad \kappa_{b}=1.06_{-0.18}^{+0.19} .
$$

The quoted results are in full agreement with the bounds obtained by CMS [41], and yield clear and model-independent evidence for the existence of non-zero top-quark and bottom-quark Yukawa couplings in nature. Despite significant experimental and theoretical effort [42-62] only very weak (no relevant) bounds exist at present in the case of the secondgeneration (first-generation) quarks. Whether the Higgs mechanism is responsible for the generation of the masses of the charm, strange, down and up quark is thus an open question, and new-physics scenarios (see e.g. [63]) that predict a significant reduction of the couplings of the observed Higgs boson to the first two generation of quarks, i.e. $\kappa_{c, s, d, u} \simeq 0$, are from the phenomenological point of view a viable option.

A question that one therefore may want to ask is how sensitively the LHC limits [5-8] and the EDM constraints [30, 31] depend on the assumption that the observed Higgs boson

\footnotetext{
${ }^{1}$ CP-violating diagonal [13, 23] and flavour-changing Higgs-fermion [15] couplings involving the third generation would be subject to stringent EDM constraints.

${ }^{2}$ In the considered benchmark model no new-physics contributions to Higgs-boson decays are assumed to exist and Higgs-boson vertices involving loops are resolved in terms of their SM content.
} 

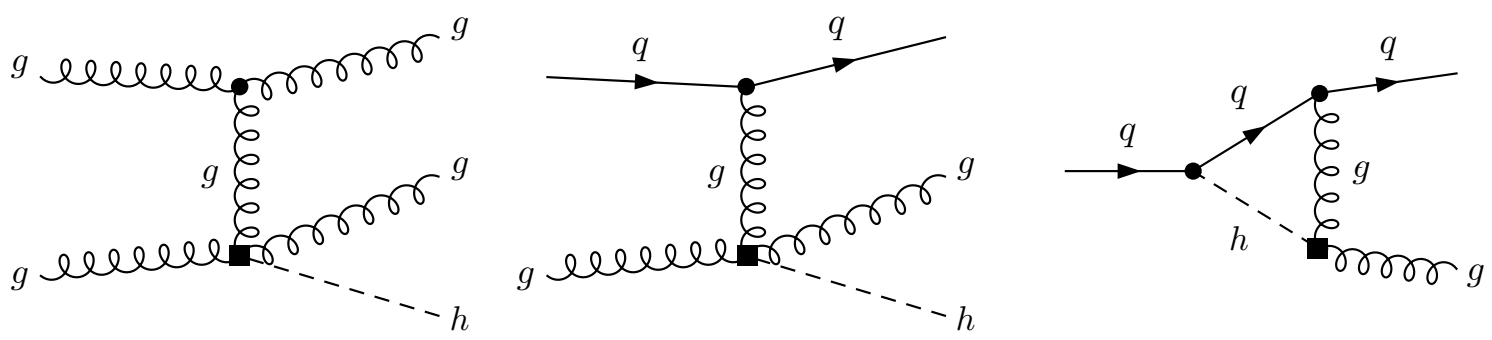

Figure 1. Left: tree-level graphs that give rise to $p p \rightarrow h+2 j$ production at the LHC. Right: example one-loop diagram that contributes for instance to the nEDM. The black squares indicate insertions of the CP-violating dimension-six operator (1.1).

has couplings to the light fermions. In the case of the operator (1.1) this question can be answered immediately by looking at the Feynman diagrams depicted in figure 1 . The two tree-level diagrams on the left-hand side give a correction to Higgs plus two jet production $p p \rightarrow h+2 j$, while the one-loop graph shown on the right induces chromoelectric dipole moments (CEDMs) for light quarks, which in turn generate non-zero contributions to the neutron EDM (nEDM) and all other hadronic EDMs. Since the diagrams that lead to the LHC signal do not involve a vertex where the Higgs couples to a light quark, the constraints on $C_{\phi \tilde{G}}$ that can be obtained from kinematic properties of $p p \rightarrow h+2 j$ are obviously independent of the size of the $\kappa_{q}$ parameters. The amplitude of the chromoelectric dipole transition $q \rightarrow q g$ instead depends linearly on $\kappa_{q}$, and hence tends to zero in the limit of vanishing light-quark Yukawa couplings. In consequence, if the down-quark and up-quark Yukawa couplings are identical to zero, no bound on the Wilson coefficient $C_{\phi \tilde{G}}$ can be obtained from hadronic EDM searches at the one-loop level. The same statement can be shown to hold for the Wilson coefficients of the dimension-six operators which encode the CP-violating couplings between the Higgs and EW gauge bosons and contribute to the eEDM. Since in $[30,31]$ it is assumed that the Yukawa couplings of light fermions are exactly SM-like, it follows that the limits derived in these papers do not directly apply in the case that the light-fermion Yukawa couplings vanish exactly or are strongly suppressed.

Motivated by the above observation, we compute in section 2 of this article the leading contributions to the nEDM that involve an insertion of (1.1) and that survive in the limit of vanishing light-quark Yukawa couplings. An extension of our calculation to the case of the full set of dimension-six operators that encode the CP-violating interactions between the Higgs and EW gauge bosons will be presented elsewhere [64]. Based on our results, we then derive in section 3 the bounds on the Wilson coefficient of the CP-violating dimensionsix operator (1.1) from current and future nEDM searches, comparing our findings to the existing LHC limits and their projections. In appendix A we extend the formalism presented in section 2.1 to the case where the light-quark Yukawa couplings are SM-like.

\section{Calculation}

Before describing the basic steps of our calculation, we mention that after EWSB the operator (1.1) shifts the QCD $\theta$ term $\mathcal{L}_{\theta}=g_{s}^{2} /\left(32 \pi^{2}\right) \theta \tilde{G}_{\mu \nu}^{a} G^{a \mu \nu}$ by a finite amount, i.e. 
$\theta \rightarrow \theta-16 \pi^{2} v^{2} C_{\phi \tilde{G}}$. Since based on the current experimental nEDM results $[65,66]$ one has $|\theta| \lesssim 10^{-10}$, we will assume that the total $\theta$ term vanishes dynamically due to a PecceiQuinn mechanism [67]. Under this assumption there is no direct bound on (1.1), and the Wilson coefficient $C_{\phi \tilde{G}}$ can be treated as a free parameter in the SMEFT as done in the analyses [5-8, 31].

As explained at the end of section 1, the goal of this work is it to calculate the numerically most relevant contributions to the nEDM that are proportional to the Wilson coefficient $C_{\phi \tilde{G}}$ and that involve the Yukawa couplings of the third-generation quarks. It turns out that at the matching scale the relevant loop graphs give rise to the following two CP-violating higher-dimensional operators of Weinberg type [68-71]

$$
\mathcal{L}_{W 1}=-\frac{g_{s}}{3} f^{a b c} \tilde{G}_{\mu \nu}^{a} G^{b \nu \rho} G_{\rho}^{c \mu} C_{3 \tilde{G}}-\frac{g_{s}^{2}}{12} \tilde{G}_{\mu \nu}^{a} G^{a \mu \nu} G_{\rho \lambda}^{b} G^{b \rho \lambda} C_{4 \tilde{G}, 1},
$$

where $f^{a b c}$ are the fully anti-symmetric structure constants of $\mathrm{SU}(3)$ colour. Since the bottom-quark contributions can be shown to be suppressed relative to the top-quark effects by a factor of $m_{b}^{2} / m_{h}^{2} \ln ^{2}\left(m_{b}^{2} / m_{h}^{2}\right) \simeq 5 \%$, we neglect corrections that are proportional to the bottom-quark Yukawa coupling in what follows. The top-quark effects are all linearly dependent on the coupling modifier $\kappa_{t}$. In view of the observed SM-like nature (1.4) of the top-quark Yukawa coupling, we will simply employ $\kappa_{t}=1$ in our calculations. Allowing for $\mathcal{O}(10 \%)$ variations of $\kappa_{t}$ would, however, not qualitatively change the results of our numerical analysis performed in section 3 .

\subsection{Dimension-six contribution}

The leading-order ( $\mathrm{LO})$ matching correction to the Wilson coefficient $C_{3 \tilde{G}}$ proportional to $C_{\phi \tilde{G}}$ arises from two-loop Feynman diagrams like the ones displayed on the left-hand side in figure 2. Employing a hard mass procedure (see [72] for a review) to obtain systematic expansions of the relevant two-loop diagrams in powers of the external momenta and the ratio $x=m_{t}^{2} / m_{h}^{2}$ with $m_{t} \simeq 163 \mathrm{GeV}$ and $m_{h} \simeq 125 \mathrm{GeV}$ the top-quark and Higgs-boson mass, we find the following analytic result

$$
C_{3 \tilde{G}}\left(m_{h}\right)=\frac{\alpha_{s}^{2}\left(m_{h}\right)}{8 \pi^{2}}\left[\frac{65}{6}+2 \ln x+\frac{1}{x}\left(\frac{383}{900}+\frac{2}{15} \ln x\right)\right] C_{\phi \tilde{G}}\left(m_{h}\right),
$$

where $\alpha_{s}=g_{s}^{2} /(4 \pi)$. The expression given above corresponds to the $\overline{\mathrm{MS}}$ scheme with the renormalisation scale set to $\mu=m_{h} \cdot{ }^{3}$ The actual calculation was performed in a $R_{\xi}$ background field gauge for the gluon [74, 75] keeping an arbitrary gauge parameter. The Levi-Civita tensor $\epsilon_{\mu \nu \rho \lambda}$ was treated as an external four-dimensional object. Our computations made use of the in-house codes that were developed in the context of [76,77], except for the tensor reduction of two-point and three-point one-loop integrals which relied on Package-X [78]. We add that we have calculated higher-order terms in the $1 / x$ expansion

\footnotetext{
${ }^{3}$ The sum of the bare two-loop Feynman diagrams that contributes to (2.2) is not ultraviolet (UV) finite. The remaining UV pole is cancelled by taking into account the one-loop mixing of the operator $\phi^{\dagger} \phi G_{\mu \nu}^{a} \tilde{G}^{a \mu \nu}$ into $\bar{Q}_{L} \sigma^{\mu \nu} t^{a} u_{R} \tilde{\phi} G_{\mu \nu}^{a}+$ h.c. with $t^{a}$ denoting the generator of SU(3) colour. We have calculated the relevant one-loop mixing finding agreement with the result given in [73].
} 

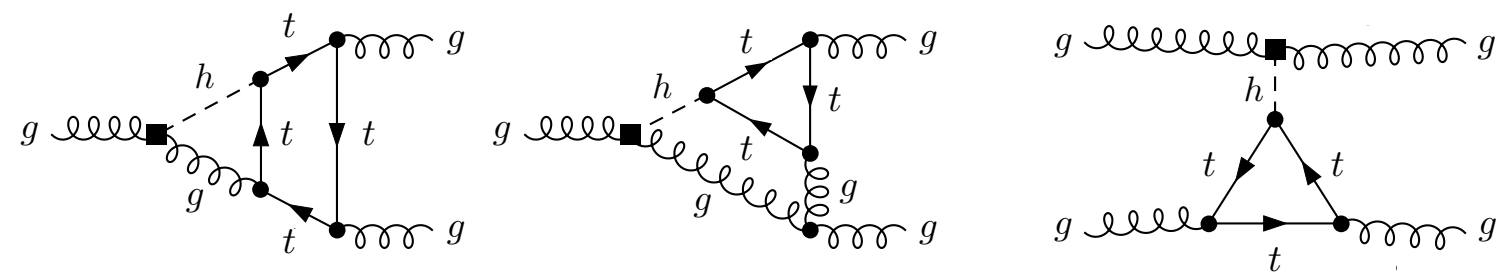

Figure 2. Left: example diagrams of two-loop corrections to $C_{3 \tilde{G}}$ arising from the insertion of $C_{\phi \tilde{G}}$. Right: a one-loop correction to $C_{4 \tilde{G}, 1}$ arising from the insertion of $C_{\phi \tilde{G}}$. The operator insertions are indicated by black squares.

of $C_{3 \tilde{G}}\left(m_{h}\right)$ and found that these corrections shift the numerical value of the matching correction (2.2) by less than a permille. ${ }^{4}$ Such an accuracy is more than sufficient for our purpose.

The renormalisation group (RG) flow from the EW to the hadronic scale $\mu_{H}=1 \mathrm{GeV}$ does not only change the value of the Wilson coefficient $C_{3 \tilde{G}}$, but also induces non-zero contributions for the EDMs $d_{q}$ and the CEDMs $\tilde{d}_{q}$ of the down and up quarks

$$
\mathcal{L}_{d}=-\frac{i}{2} \bar{q} \sigma_{\mu \nu} \gamma_{5} q F^{\mu \nu} d_{q}-\frac{i}{2} g_{s} \bar{q} \sigma_{\mu \nu} t^{a} \gamma_{5} q G^{a \mu \nu} \tilde{d}_{q},
$$

with $q=d, u, \sigma^{\mu \nu}=i / 2\left(\gamma^{\mu} \gamma^{\nu}-\gamma^{\mu} \gamma^{\nu}\right)$, and $F_{\mu \nu}$ denotes the QED field strength tensor. In the basis $\vec{C}_{6}=\left(d_{q}, \tilde{d}_{q}, C_{3 \tilde{G}}\right)^{T}$, the one-loop anomalous dimension (AD) matrix takes the following form [79-81]

$$
\hat{\gamma}_{6}=\left(\begin{array}{ccc}
\frac{32}{3} & 0 & 0 \\
\frac{32}{3} & \frac{28}{3} & 0 \\
0 & -6 & 3+2 N_{F}+2 \beta_{0}
\end{array}\right) \text {, }
$$

where $\beta_{0}=11-2 / 3 N_{F}$ is the LO QCD beta function and $N_{F}$ denotes the number of active quark flavours. Resumming leading-logarithmic (LL) corrections in the five-flavour, four-flavour and three-flavour theory, we obtain

$$
\begin{aligned}
d_{q}\left(\mu_{H}\right) & \simeq-5.6 \cdot 10^{-2} e Q_{q} m_{q}\left(\mu_{H}\right) C_{3 \tilde{G}}\left(m_{h}\right), \\
\tilde{d}_{q}\left(\mu_{H}\right) & \simeq 1.2 \cdot 10^{-1} m_{q}\left(\mu_{H}\right) C_{3 \tilde{G}}\left(m_{h}\right), \\
C_{3 \tilde{G}}\left(\mu_{H}\right) & \simeq 1.3 \cdot 10^{-1} C_{3 \tilde{G}}\left(m_{h}\right) .
\end{aligned}
$$

Here $e$ denotes the electron charge magnitude, $Q_{q}$ is the fractional electric charge of the relevant quark and $m_{q}\left(\mu_{H}\right)$ is its $\overline{\mathrm{MS}}$ mass at the hadronic scale. The numerical factors in (2.5) correspond to the values $\alpha_{s}\left(m_{h}\right) \simeq 0.11, \alpha_{s}\left(m_{b}\right) \simeq 0.21, \alpha_{s}\left(m_{c}\right) \simeq 0.32$ and $\alpha_{s}\left(\mu_{H}\right) \simeq 0.36$ of the $\mathrm{QCD}$ coupling constant. Notice that the Wilson coefficient $C_{3 \tilde{G}}$

\footnotetext{
${ }^{4}$ The analytic expressions for the $\mathcal{O}\left(1 / x^{2}\right)$ and $\mathcal{O}\left(1 / x^{3}\right)$ terms can be found in the $\mathrm{L}_{\mathrm{A}}^{\mathrm{A} X}$ source code of this article.
} 
of the dimension-six Weinberg operator gets strongly suppressed by one-loop RG running in QCD. ${ }^{5}$

The hadronic matrix elements of the dimension-six operators corresponding to the Wilson coefficients $d_{q}, \tilde{d}_{q}$ and $C_{3 \tilde{G}}$ in (2.5) are known with varying levels of theoretical uncertainties. The EDM contributions from down and up quarks have been calculated with an accuracy of $5 \%$ using lattice QCD (LQCD) [86-88], while QCD sum-rule calculations [9, 89-91] allow to determine the contributions from the down-quark and up-quark CEDMs with uncertainties of $50 \%$. At present only estimates of the hadronic matrix element of the dimension-six Weinberg operator exist that rely on either QCD sum rules [92, 93], the vacuum insertion approximation [94] or naive dimensional analysis [68]. Since only the QCD sum-rules calculations allow for a systematic analysis of theoretical uncertainties, we will in the following rely on them. Adopting our very recent QCD sum-rule estimate [93], which is plagued by an uncertainty of $50 \%$, and employing $m_{d}\left(\mu_{H}\right)=5.4 \cdot 10^{-3} \mathrm{GeV}$ and $m_{u}\left(\mu_{H}\right)=2.5 \cdot 10^{-3} \mathrm{GeV}[95]$, we obtain

$$
\frac{\left(d_{n}\right)_{3 \tilde{G}}}{e}=[1.0(1 \pm 0.05)+8.8(1 \pm 0.5)-66.6(1 \pm 0.5)] C_{3 \tilde{G}}\left(m_{h}\right) \cdot 10^{-4} \mathrm{GeV}
$$

where the first, second and third term corresponds to the $d_{q}, \tilde{d}_{q}$ and $C_{3 \tilde{G}}$ contribution in (2.5), respectively. We emphasise that in the case that new physics enters only through the matching correction $C_{3 \tilde{G}}\left(m_{h}\right)$ the relative signs in (2.6) are all fixed, meaning that the contribution from the dimension-six Weinberg operator necessarily interferes destructively with both the EDM and CEDM contribution.

\subsection{Dimension-eight contribution}

At LO the matching correction to the Wilson coefficient $C_{4 \tilde{G}, 1}$ proportional to $C_{\phi \tilde{G}}$ arise from one-loop graphs of the type shown on the right in figure 2. A straightforward calculation gives

$$
C_{4 \tilde{G}, 1}\left(m_{h}\right)=\frac{\alpha_{s}\left(m_{h}\right)}{\pi} \frac{1}{m_{h}^{2}}\left[1+\frac{7}{120 x}+\frac{1}{168 x^{2}}\right] C_{\phi \tilde{G}}\left(m_{h}\right),
$$

at the matching scale $\mu=m_{h}$. Higher-order terms in the $1 / x$ expansion change the matching correction $C_{4 \tilde{G}, 1}\left(m_{h}\right)$ by less than a permille, and we have therefore not included them in $(2.7) .{ }^{6}$

At the dimension-eight level there are three independent CP-violating operators that can be built from QCD field strength tensors (see [69-71] for details). While only the operator appearing in (2.1) receives a one-loop matching correction proportional to $C_{\phi \tilde{G}}$ all

\footnotetext{
${ }^{5}$ The two-loop and three-loop $N_{F}$-independent contributions to the AD of the dimension-six Weinberg operator have been calculated very recently [82]. Due to cancellations between the next-to-leading-logarithmic and the next-to-next-to-leading-logarithmic QCD corrections, the total three-loop result is numerically close to the one-loop result for $C_{3 \tilde{G}}\left(\mu_{H}\right)$ reported in (2.5). In view of this and given the sizeable uncertainties of the hadronic matrix element of the dimension-six Weinberg operator (cf. (2.6)) using only the LL RG evolution is in our opinion fully justified. In the same spirit, the two-loop and three-loop mixing of the quark EDMs and CEDMs [81, 83-85] is also neglected in (2.5).

${ }^{6}$ The results for the $\mathcal{O}\left(1 / x^{3}\right)$ and $\mathcal{O}\left(1 / x^{4}\right)$ terms of $(2.7)$ can be found in the ${ }^{\mathrm{A}} \mathrm{TEX}$ source code of this paper.
} 
three dimension-eight operators mix under QCD. We write the two additional CP-violating four-gluon operators as

$$
\mathcal{L}_{W 2}=-\frac{g_{s}^{2}}{12} \tilde{G}_{\mu \nu}^{a} G^{b \mu \nu} G_{\rho \lambda}^{a} G^{b \rho \lambda} C_{4 \tilde{G}, 2}-\frac{g_{s}^{2}}{12} d_{a b e} d_{c d e} \tilde{G}_{\mu \nu}^{a} G^{b \mu \nu} G_{\rho \lambda}^{c} G^{d \rho \lambda} C_{4 \tilde{G}, 3}
$$

where $d_{a b c}$ are totally symmetric structure constants of QCD. In the basis $\vec{C}_{8}=\left(C_{4 \tilde{G}, 1}\right.$, $\left.C_{4 \tilde{G}, 2}, C_{4 \tilde{G}, 3}\right)^{T}$ the one-loop AD matrix then reads [69-71]

$$
\hat{\gamma}_{8}=\left(\begin{array}{ccc}
-56+\frac{8}{3} N_{F}+2 \beta_{0} & 24 & -36 \\
-38 & 56+\frac{8}{3} N_{F}+2 \beta_{0} & -42 \\
-14 & 12 & -14+\frac{8}{3} N_{F}+2 \beta_{0}
\end{array}\right) .
$$

Working in the five, four and three flavour theory and using the values of the QCD coupling constant given earlier leads to the LL approximations

$$
\begin{aligned}
& C_{4 \tilde{G}, 1}\left(\mu_{H}\right) \simeq 6.6 C_{4 \tilde{G}, 1}\left(m_{h}\right), \\
& C_{4 \tilde{G}, 2}\left(\mu_{H}\right) \simeq-1.9 C_{4 \tilde{G}, 1}\left(m_{h}\right), \\
& C_{4 \tilde{G}, 3}\left(\mu_{H}\right) \simeq 3.9 C_{4 \tilde{G}, 1}\left(m_{h}\right) .
\end{aligned}
$$

Notice that in contrast to (2.5) the Wilson coefficient $C_{4 \tilde{G}, 1}$ of the dimension-eight Weinberg operator that is generated at the matching scale gets enhanced by RG running.

Estimates of the hadronic matrix elements of the dimension-eight CP-violating fourgluon operators have very recently been obtained in the context of QCD sum rules [93]. Employing these results we find

$$
\frac{\left(d_{n}\right)_{4 \tilde{G}}}{e}=-6.1(1 \pm 0.8) C_{4 \tilde{G}, 1}\left(m_{h}\right) \cdot 10^{-1} \mathrm{GeV}^{3},
$$

which has a theoretical uncertainty of $80 \%$. We add that the sign in $\left(d_{n}\right)_{4 \tilde{G}} / e$ is predicted in the QCD sum-rule approach and that neglecting all contributions from the two additional dimension-eight operators (2.8) would lead to a numerical result that deviates from (2.11) by less than $5 \%$.

\section{Discussion}

To discuss the constraints that nEDM measurements can set on the CP-violating dimension-six Higgs-gluon interactions appearing in (2.1), we introduce the dimensionless Wilson coefficient

$$
\bar{C}_{\phi \tilde{G}}\left(m_{h}\right)=v^{2} C_{\phi \tilde{G}}\left(m_{h}\right) .
$$

Combining (2.6) and (2.11) we then find in terms of the bared Wilson coefficient the following expression

$$
\left|\frac{d_{n}}{e}\right|=6.2|(1 \pm 0.05)+8.6(1 \pm 0.5)-65.1(1 \pm 0.5)-7.5(1 \pm 0.8)|\left|\bar{C}_{\phi \tilde{G}}\left(m_{h}\right)\right| \cdot 10^{-26} \mathrm{~cm},
$$


where the contributions associated to the terms $d_{q}, \tilde{d}_{q}, C_{3 \tilde{G}}$ and the dimension-eight Weinberg operators have been kept distinct.

In view of the sizeable hadronic uncertainties of the matrix elements of the operators of CEDM and Weinberg type and the relative overall sign of the Weinberg-type contributions, we combine the errors in (3.2) in such a way that our prediction

$$
\left|\frac{d_{n}}{e}\right|=1.3\left|\bar{C}_{\phi \tilde{G}}\left(m_{h}\right)\right| \cdot 10^{-24} \mathrm{~cm}
$$

provides a lower absolute limit on the actual size of the $\bar{C}_{\phi \tilde{G}}$ corrections to $d_{n}$. Because our error treatment assumes a cancellation between the numerically dominant contributions associated to $C_{3 \tilde{G}}, \tilde{d}_{q}$ and the dimension-eight terms, it is conservative. Notice that if such a cancellation is not at work in practice, predictions for $\left|d_{n} / e\right|$ can be obtained that are larger by a factor of about 5 than the upper limit (3.3) but still consistent within the individual uncertainties quoted in (3.2).

The current experimental nEDM results $[65,66]$ impose the following $95 \%$ CL bound

$$
\left|\frac{d_{n}}{e}\right|<3.6 \cdot 10^{-26} \mathrm{~cm}
$$

which, using the lower limit of the only quark EDMs case in (3.3) translates into

$$
\left|\bar{C}_{\phi \tilde{G}}\left(m_{h}\right)\right|<2.9 \cdot 10^{-2} .
$$

As shown in appendix A, this bound is weaker by a factor of almost 30 than the $95 \%$ CL exclusion obtained in universal theories that assume that the Yukawa couplings of the light quarks are SM-like. The constraint (3.5) can also be compared to the 95\% CL limit

$$
\bar{C}_{\phi \tilde{G}}\left(m_{h}\right) \in[-0.13,0.83] \cdot 10^{-2}
$$

on the dimensionless Wilson coefficient (3.1) that has been obtained in [8] from an analysis of the azimuthal angle difference $\Delta \phi_{j j}$ between the two jets in $h+2 j$ LHC events.

In order to obtain an idea of the prospects of the low-energy constraints, we assume that a lower bound of

$$
\left|\frac{d_{n}}{e}\right|<1.0 \cdot 10^{-27} \mathrm{~cm}
$$

can be set at the proposed PSI and LANL nEDM experiments [96, 97]. In such a case, one arrives at the limit

$$
\left|\bar{C}_{\phi \tilde{G}}\left(m_{h}\right)\right|<8.0 \cdot 10^{-4}
$$

if one assumes that (3.3) provides a lower absolute limit on the $\bar{C}_{\phi \tilde{G}}$ corrections to $d_{n}$. The sensitivity study [8] of the $p p \rightarrow h+2 j$ process finds on the other hand that the high-luminosity LHC (HL-LHC) should be able to set a bound of

$$
\left|\bar{C}_{\phi \tilde{G}}\left(m_{h}\right)\right|<9.2 \cdot 10^{-4} .
$$

A comparison of the limits (3.5) and (3.6) on the CP-violating interactions involving the Higgs boson and gluons shows that at present the sensitivity of nEDM searches is by 
roughly an order of magnitude weaker than the constraining power of the LHC. This is mainly a result of the conservative treatment of the hadronic uncertainties in (3.2) that led to (3.3). From (3.8) and (3.9) it is however also evident that in the future the sensitivity of nEDM searches can reach the LHC level even if the accuracy of the hadronic matrix elements are not improved. First-principle calculations of the matrix elements of the CEDMs and the dimension-six Weinberg operator are possible using existing LQCD methodology, and considering the efforts by several LQCD groups [98-105], it seems possible that estimates with uncertainties similar to the current ones can be obtained within the next five years $[106,107]$. It remains to be seen which accuracy such computations can achieve in the next 20 years of LHC running, but we believe that it is very likely that the bound (3.8) can be improved by the end of the HL-LHC run.

From the above discussion one can conclude that future nEDM searches and LHC measurements are complementary to each other even in the specific class of new-physics models where the Yukawa couplings of light quarks are zero. Since such new-physics realisations represent in some sense the worst-case scenario for the low-energy constraints considered here, we believe this to be an interesting finding. Our results show furthermore that in a global SMEFT analysis, EDM constraints can have additional flat or weakly bound directions that do not appear in the case of the class of universal theories considered in [30, 31]. As already emphasised in [31], to resolve unbounded directions in the multi-dimensional space of Wilson coefficients, high- $p_{T}$ and low-energy constraints on CP-violating couplings between the Higgs and gauge bosons should be combined into global fits. The nEDM results presented in this article can be readily used for such a purpose.

\section{Acknowledgments}

The Feynman diagrams shown in this article and the analytic expression for the relevant one-loop and two-loop scattering amplitudes have been obtained using FeynArts [108]. The Feynman rules for the higher-dimensional operators (1.1) and (2.1) have been derived with the help of the FeynRules package [109].

\section{A Case of universal theories}

In this appendix we extend the formalism presented in section 2.1 to the case of universal theories. Under the assumption that the light-quark Yukawa couplings take the values predicted in the SM (i.e. $\kappa_{q}=1$ ) the one-loop diagram on the right-hand side in figure 1 induces a CEDM for the down and up quark. In agreement with [31], we find the following expression for the one-loop correction to the CEDMs

$$
\tilde{d}_{q}\left(m_{h}\right)=\frac{3 \alpha_{s}\left(m_{h}\right)}{2 \pi} C_{\phi \tilde{G}}\left(m_{h}\right),
$$

at the matching scale $\mu=m_{h}$. Including the contribution (A.1) in the evaluation of the nEDM, the formula (2.6) turns into

$$
\begin{aligned}
\frac{\left(d_{n}\right)_{\tilde{d}_{q}, 3 \tilde{G}}}{e}=[ & \left(6.9 \tilde{d}_{q}\left(m_{h}\right)+1.0 C_{3 \tilde{G}}\left(m_{h}\right)\right)(1 \pm 0.05)+\left(36.7 \tilde{d}_{q}\left(m_{h}\right)+8.8 C_{3 \tilde{G}}\left(m_{h}\right)\right)(1 \pm 0.5) \\
& \left.-66.6 C_{3 \tilde{G}}\left(m_{h}\right)(1 \pm 0.5)\right] \cdot 10^{-4} \mathrm{GeV} .
\end{aligned}
$$


Adding the new dimension-six contribution (A.2) to the dimension-eight piece (2.11) we then obtain the formula

$$
\begin{aligned}
\left|\frac{d_{n}}{e}\right|=6.2 \mid & 190.6(1 \pm 0.05)+1016.6(1 \pm 0.5) \\
& -65.1(1 \pm 0.5)-7.5(1 \pm 0.8)|| \bar{C}_{\phi \tilde{G}}\left(m_{h}\right) \mid \cdot 10^{-26} \mathrm{~cm}
\end{aligned}
$$

where $\bar{C}_{\phi \tilde{G}}$ denotes the dimensionless Wilson coefficient introduced in (3.1). In view of the sizeable hadronic uncertainties of the matrix elements in (A.3) and the relative overall sign of the Weinberg-type contributions, we again combine the errors in $\left|d_{n} / e\right|$ in such a way that our final prediction provides a lower absolute limit on the actual size of the $\bar{C}_{\phi \tilde{G}}$ corrections to the nEDM. We find

$$
\left|\frac{d_{n}}{e}\right|=3.6\left|\bar{C}_{\phi \tilde{G}}\left(m_{h}\right)\right| \cdot 10^{-23} \mathrm{~cm}
$$

Numerically, the result (A.4) implies that

$$
\begin{aligned}
& \left|\frac{d_{n}}{e}\right|<3.6 \cdot 10^{-26} \mathrm{~cm} \quad \Rightarrow \quad\left|\bar{C}_{\phi \tilde{G}}\left(m_{h}\right)\right|<1.0 \cdot 10^{-3}, \\
& \left|\frac{d_{n}}{e}\right|<1.0 \cdot 10^{-27} \mathrm{~cm} \Rightarrow\left|\bar{C}_{\phi \tilde{G}}\left(m_{h}\right)\right|<2.8 \cdot 10^{-5} .
\end{aligned}
$$

Notice that the $95 \%$ CL limits on $\left|\bar{C}_{\phi \tilde{G}}\left(m_{h}\right)\right|$ as given in (A.5) are comparable to the bounds that have been derived in [31] by using the so-called Rfit strategy, in which all hadronic matrix elements entering the prediction for the nEDM are varied within their allowed ranges.

Open Access. This article is distributed under the terms of the Creative Commons Attribution License (CC-BY 4.0), which permits any use, distribution and reproduction in any medium, provided the original author(s) and source are credited.

\section{References}

[1] T. Plehn, D.L. Rainwater and D. Zeppenfeld, Determining the Structure of Higgs Couplings at the LHC, Phys. Rev. Lett. 88 (2002) 051801 [hep-ph/0105325] [INSPIRE].

[2] G. Klamke and D. Zeppenfeld, Higgs plus two jet production via gluon fusion as a signal at the CERN LHC, JHEP 04 (2007) 052 [hep-ph/0703202] [INSPIRE].

[3] W. Buchmüller and D. Wyler, Effective Lagrangian Analysis of New Interactions and Flavor Conservation, Nucl. Phys. B 268 (1986) 621 [INSPIRE].

[4] B. Grzadkowski, M. Iskrzynski, M. Misiak and J. Rosiek, Dimension-Six Terms in the Standard Model Lagrangian, JHEP 10 (2010) 085 [arXiv: 1008.4884] [INSPIRE].

[5] ATLAS collaboration, Constraints on non-Standard Model Higgs boson interactions in an effective Lagrangian using differential cross sections measured in the $H \rightarrow \gamma \gamma$ decay channel at $\sqrt{s}=8 \mathrm{TeV}$ with the ATLAS detector, Phys. Lett. B 753 (2016) 69 [arXiv:1508.02507] [INSPIRE]. 
[6] F. Ferreira, B. Fuks, V. Sanz and D. Sengupta, Probing CP-violating Higgs and gauge-boson couplings in the Standard Model effective field theory, Eur. Phys. J. C 77 (2017) 675 [arXiv: 1612.01808] [INSPIRE].

[7] ATLAS collaboration, Measurements of Higgs boson properties in the diphoton decay channel with $36 \mathrm{fb}^{-1}$ of pp collision data at $\sqrt{s}=13 \mathrm{TeV}$ with the ATLAS detector, Phys. Rev. D 98 (2018) 052005 [arXiv: 1802. 04146] [InSPIRE].

[8] F.U. Bernlochner et al., Angles on CP-violation in Higgs boson interactions, Phys. Lett. B 790 (2019) 372 [arXiv: 1808.06577] [INSPIRE].

[9] M. Pospelov and A. Ritz, Electric dipole moments as probes of new physics, Annals Phys. 318 (2005) 119 [hep-ph/0504231] [INSPIRE].

[10] Y. Li, S. Profumo and M. Ramsey-Musolf, A Comprehensive Analysis of Electric Dipole Moment Constraints on CP-violating Phases in the MSSM, JHEP 08 (2010) 062 [arXiv: 1006.1440$]$ [INSPIRE].

[11] J. Engel, M.J. Ramsey-Musolf and U. van Kolck, Electric Dipole Moments of Nucleons, Nuclei and Atoms: The Standard Model and Beyond, Prog. Part. Nucl. Phys. 71 (2013) 21 [arXiv: 1303.2371] [INSPIRE].

[12] M. Jung and A. Pich, Electric Dipole Moments in Two-Higgs-Doublet Models, JHEP 04 (2014) 076 [arXiv: 1308.6283] [INSPIRE].

[13] J. Brod, U. Haisch and J. Zupan, Constraints on CP-violating Higgs couplings to the third generation, JHEP 11 (2013) 180 [arXiv:1310.1385] [INSPIRE].

[14] S. Inoue, M.J. Ramsey-Musolf and Y. Zhang, CP-violating phenomenology of flavor conserving two Higgs doublet models, Phys. Rev. D 89 (2014) 115023 [arXiv:1403.4257] [INSPIRE].

[15] M. Gorbahn and U. Haisch, Searching for $t \rightarrow c(u) h$ with dipole moments, JHEP 06 (2014) 033 [arXiv: 1404.4873] [inSPIRE].

[16] W. Altmannshofer, J. Brod and M. Schmaltz, Experimental constraints on the coupling of the Higgs boson to electrons, JHEP 05 (2015) 125 [arXiv:1503.04830] [INSPIRE].

[17] Y.T. Chien, V. Cirigliano, W. Dekens, J. de Vries and E. Mereghetti, Direct and indirect constraints on CP-violating Higgs-quark and Higgs-gluon interactions, JHEP 02 (2016) 011 [arXiv: 1510.00725] [INSPIRE].

[18] V. Cirigliano, W. Dekens, J. de Vries and E. Mereghetti, Is there room for CP-violation in the top-Higgs sector?, Phys. Rev. D 94 (2016) 016002 [arXiv: 1603.03049] [INSPIRE].

[19] V. Cirigliano, W. Dekens, J. de Vries and E. Mereghetti, Constraining the top-Higgs sector of the Standard Model Effective Field Theory, Phys. Rev. D 94 (2016) 034031 [arXiv: 1605.04311] [INSPIRE].

[20] N. Yamanaka, B.K. Sahoo, N. Yoshinaga, T. Sato, K. Asahi and B.P. Das, Probing exotic phenomena at the interface of nuclear and particle physics with the electric dipole moments of diamagnetic atoms: A unique window to hadronic and semi-leptonic CP-violation, Eur. Phys. J. A 53 (2017) 54 [arXiv: 1703.01570] [InSPIRE].

[21] K. Yanase, N. Yoshinaga, K. Higashiyama and N. Yamanaka, Electric dipole moment of ${ }^{199} \mathrm{Hg}$ atom from P, CP-odd electron-nucleon interaction, Phys. Rev. D 99 (2019) 075021 [arXiv: 1805.00419] [INSPIRE]. 
[22] W. Dekens, J. de Vries, M. Jung and K.K. Vos, The phenomenology of electric dipole moments in models of scalar leptoquarks, JHEP 01 (2019) 069 [arXiv:1809.09114] [INSPIRE].

[23] J. Brod and E. Stamou, Electric dipole moment constraints on CP-violating heavy-quark Yukawas at next-to-leading order, arXiv:1810.12303 [INSPIRE].

[24] J. Brod and D. Skodras, Electric dipole moment constraints on CP-violating light-quark Yukawas, JHEP 01 (2019) 233 [arXiv:1811.05480] [INSPIRE].

[25] D. McKeen, M. Pospelov and A. Ritz, Modified Higgs branching ratios versus CP and lepton flavor violation, Phys. Rev. D 86 (2012) 113004 [arXiv: 1208.4597] [InSPIRE].

[26] W.-F. Chang, W.-P. Pan and F. Xu, Effective gauge-Higgs operators analysis of new physics associated with the Higgs boson, Phys. Rev. D 88 (2013) 033004 [arXiv:1303.7035] [INSPIRE].

[27] B. Gripaios and D. Sutherland, Searches for CP-violating dimension-6 electroweak gauge boson operators, Phys. Rev. D 89 (2014) 076004 [arXiv: 1309.7822] [INSPIRE].

[28] S. Dwivedi, D.K. Ghosh, B. Mukhopadhyaya and A. Shivaji, Constraints on CP-violating gauge-Higgs operators, Phys. Rev. D 92 (2015) 095015 [arXiv: 1505.05844] [INSPIRE].

[29] C. Cesarotti, Q. Lu, Y. Nakai, A. Parikh and M. Reece, Interpreting the Electron EDM Constraint, JHEP 05 (2019) 059 [arXiv: 1810.07736] [INSPIRE].

[30] G. Panico, A. Pomarol and M. Riembau, EFT approach to the electron Electric Dipole Moment at the two-loop level, JHEP 04 (2019) 090 [arXiv:1810.09413] [INSPIRE].

[31] V. Cirigliano, A. Crivellin, W. Dekens, J. de Vries, M. Hoferichter and E. Mereghetti, CP Violation in Higgs-Gauge Interactions: From Tabletop Experiments to the LHC, Phys. Rev. Lett. 123 (2019) 051801 [arXiv: 1903.03625] [INSPIRE].

[32] R. Barbieri, A. Pomarol, R. Rattazzi and A. Strumia, Electroweak symmetry breaking after LEP-1 and LEP-2, Nucl. Phys. B 703 (2004) 127 [hep-ph/0405040] [INSPIRE].

[33] B. Gripaios, A. Pomarol, F. Riva and J. Serra, Beyond the Minimal Composite Higgs Model, JHEP 04 (2009) 070 [arXiv:0902.1483] [INSPIRE].

[34] J.R. Espinosa, B. Gripaios, T. Konstandin and F. Riva, Electroweak Baryogenesis in Non-minimal Composite Higgs Models, JCAP 01 (2012) 012 [arXiv:1110.2876] [INSPIRE].

[35] G. D'Ambrosio, G.F. Giudice, G. Isidori and A. Strumia, Minimal flavor violation: An Effective field theory approach, Nucl. Phys. B 645 (2002) 155 [hep-ph/0207036] [INSPIRE].

[36] CMS collaboration, Observation of $t \bar{t} H$ production, Phys. Rev. Lett. 120 (2018) 231801 [arXiv: 1804.02610] [INSPIRE].

[37] ATLAS collaboration, Observation of Higgs boson production in association with a top quark pair at the LHC with the ATLAS detector, Phys. Lett. B 784 (2018) 173 [arXiv: 1806.00425] [INSPIRE].

[38] ATLAS collaboration, Observation of $H \rightarrow b \bar{b}$ decays and VH production with the ATLAS detector, Phys. Lett. B 786 (2018) 59 [arXiv:1808.08238] [INSPIRE].

[39] CMS collaboration, Observation of Higgs boson decay to bottom quarks, Phys. Rev. Lett. 121 (2018) 121801 [arXiv: 1808.08242] [INSPIRE].

[40] ATLAS collaboration, Combined measurements of Higgs boson production and decay using up to $80 \mathrm{fb}^{-1}$ of proton-proton collision data at $\sqrt{\mathrm{s}}=13 \mathrm{TeV}$ collected with the ATLAS experiment, ATLAS-CONF-2019-005 (2019) [INSPIRE]. 
[41] CMS collaboration, Combined measurements of Higgs boson couplings in proton-proton collisions at $\sqrt{s}=13$ TeV, Eur. Phys. J. C 79 (2019) 421 [arXiv:1809.10733] [InSPIRE].

[42] G.T. Bodwin, F. Petriello, S. Stoynev and M. Velasco, Higgs boson decays to quarkonia and

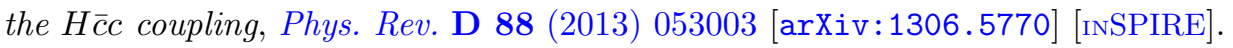

[43] C. Delaunay, T. Golling, G. Perez and Y. Soreq, Enhanced Higgs boson coupling to charm pairs, Phys. Rev. D 89 (2014) 033014 [arXiv:1310.7029] [INSPIRE].

[44] A.L. Kagan, G. Perez, F. Petriello, Y. Soreq, S. Stoynev and J. Zupan, Exclusive Window onto Higgs Yukawa Couplings, Phys. Rev. Lett. 114 (2015) 101802 [arXiv:1406.1722] [INSPIRE].

[45] ATLAS collaboration, Search for Higgs and $Z$ Boson Decays to $J / \psi \gamma$ and $\Upsilon(n S) \gamma$ with the ATLAS Detector, Phys. Rev. Lett. 114 (2015) 121801 [arXiv:1501.03276] [INSPIRE].

[46] G. Perez, Y. Soreq, E. Stamou and K. Tobioka, Constraining the charm Yukawa and Higgs-quark coupling universality, Phys. Rev. D 92 (2015) 033016 [arXiv:1503.00290] [INSPIRE].

[47] M. König and M. Neubert, Exclusive Radiative Higgs Decays as Probes of Light-Quark Yukawa Couplings, JHEP 08 (2015) 012 [arXiv: 1505.03870] [INSPIRE].

[48] G. Perez, Y. Soreq, E. Stamou and K. Tobioka, Prospects for measuring the Higgs boson coupling to light quarks, Phys. Rev. D 93 (2016) 013001 [arXiv:1505.06689] [INSPIRE].

[49] I. Brivio, F. Goertz and G. Isidori, Probing the Charm Quark Yukawa Coupling in Higgs + Charm Production, Phys. Rev. Lett. 115 (2015) 211801 [arXiv:1507.02916] [INSPIRE].

[50] F. Bishara, U. Haisch, P.F. Monni and E. Re, Constraining Light-Quark Yukawa Couplings from Higgs Distributions, Phys. Rev. Lett. 118 (2017) 121801 [arXiv:1606.09253] [INSPIRE].

[51] Y. Soreq, H.X. Zhu and J. Zupan, Light quark Yukawa couplings from Higgs kinematics, JHEP 12 (2016) 045 [arXiv: 1606.09621] [INSPIRE].

[52] F. Yu, Phenomenology of Enhanced Light Quark Yukawa Couplings and the $W^{ \pm} h$ Charge Asymmetry, JHEP 02 (2017) 083 [arXiv: 1609.06592] [INSPIRE].

[53] T. Han and X. Wang, Radiative Decays of the Higgs Boson to a Pair of Fermions, JHEP 10 (2017) 036 [arXiv:1704.00790] [INSPIRE].

[54] J. Cohen, S. Bar-Shalom, G. Eilam and A. Soni, Light-quarks Yukawa couplings and new physics in exclusive high-p $p_{T}$ Higgs boson + jet and Higgs boson + b-jet events, Phys. Rev. D 97 (2018) 055014 [arXiv:1705.09295] [INSPIRE].

[55] ATLAS collaboration, Search for the Decay of the Higgs Boson to Charm Quarks with the ATLAS Experiment, Phys. Rev. Lett. 120 (2018) 211802 [arXiv:1802.04329] [InSPIRE].

[56] CMS collaboration, Sensitivity projections for Higgs boson properties measurements at the HL-LHC, CMS-PAS-FTR-18-011 (2018) [INSPIRE].

[57] CMS collaboration, Measurement and interpretation of differential cross sections for Higgs boson production at $\sqrt{s}=13$ TeV, Phys. Lett. B 792 (2019) 369 [arXiv:1812.06504] [INSPIRE].

[58] T. Han, B. Nachman and X. Wang, Charm-quark Yukawa Coupling in $h \rightarrow c \bar{c} \gamma$ at LHC, Phys. Lett. B 793 (2019) 90 [arXiv:1812.06992] [InSPIRE]. 
[59] ATLAS collaboration, Measurements and interpretations of Higgs-boson fiducial cross sections in the diphoton decay channel using $139 \mathrm{fb}^{-1}$ of pp collision data at $\sqrt{\mathrm{s}}=13 \mathrm{TeV}$ with the ATLAS detector, ATLAS-CONF-2019-029 (2019) [INSPIRE].

[60] CMS collaboration, Search for the standard model Higgs boson decaying to charm quarks, CMS-PAS-HIG-18-031 (2019) [INSPIRE].

[61] CMS collaboration, Search for Higgs and $Z$ boson decays to $J / \psi$ or $\Upsilon$ pairs in the four-muon final state in proton-proton collisions at $\sqrt{s}=13 \mathrm{TeV}$, Phys. Lett. B 797 (2019) 134811 [arXiv: 1905.10408] [INSPIRE].

[62] L. Alasfar, R. Corral Lopez and R. Gröber, Probing Higgs couplings to light quarks via Higgs pair production, arXiv: 1909.05279 [INSPIRE].

[63] D. Ghosh, R.S. Gupta and G. Perez, Is the Higgs Mechanism of Fermion Mass Generation a Fact? A Yukawa-less First-Two-Generation Model, Phys. Lett. B 755 (2016) 504 [arXiv: 1508.01501] [INSPIRE].

[64] U. Haisch and A. Hala, in preparation.

[65] C.A. Baker et al., An Improved experimental limit on the electric dipole moment of the neutron, Phys. Rev. Lett. 97 (2006) 131801 [hep-ex/0602020] [INSPIRE].

[66] J.M. Pendlebury et al., Revised experimental upper limit on the electric dipole moment of the neutron, Phys. Rev. D 92 (2015) 092003 [arXiv:1509.04411] [InSPIRE].

[67] R.D. Peccei and H.R. Quinn, CP Conservation in the Presence of Instantons, Phys. Rev. Lett. 38 (1977) 1440 [INSPIRE].

[68] S. Weinberg, Larger Higgs Exchange Terms in the Neutron Electric Dipole Moment, Phys. Rev. Lett. 63 (1989) 2333 [INSPIRE].

[69] A.Y. Morozov, Matrix Of Mixing Of Scalar And Vector Mesons Of Dimension D $\leq 8$ In QCD (in Russian), Sov. J. Nucl. Phys. 40 (1984) 505 [Yad. Fiz. 40 (1984) 788] [InSPIRE].

[70] D. Chang, T.W. Kephart, W.-Y. Keung and T.C. Yuan, The Chromoelectric dipole moment of the heavy quark and purely gluonic CP-violating operators, Phys. Rev. Lett. 68 (1992) 439 [INSPIRE].

[71] M.J. Booth, Anomalous dimensions of Weinberg operators, Phys. Rev. D 45 (1992) 2518 [INSPIRE].

[72] V.A. Smirnov, Applied asymptotic expansions in momenta and masses, Springer Tracts Mod. Phys. 177 (2002) 1 [inSPIRE].

[73] R. Alonso, E.E. Jenkins, A.V. Manohar and M. Trott, Renormalization Group Evolution of the Standard Model Dimension Six Operators III: Gauge Coupling Dependence and Phenomenology, JHEP 04 (2014) 159 [arXiv:1312.2014] [INSPIRE].

[74] L.F. Abbott, The Background Field Method Beyond One Loop, Nucl. Phys. B 185 (1981) 189 [INSPIRE].

[75] L.F. Abbott, Introduction to the Background Field Method, Acta Phys. Polon. B 13 (1982) 33 [INSPIRE].

[76] W. Bizoń, U. Haisch and L. Rottoli, Constraints on the quartic Higgs self-coupling from double-Higgs production at future hadron colliders, JHEP 10 (2019) 267 [arXiv: 1810.04665] [INSPIRE].

[77] M. Gorbahn and U. Haisch, Two-loop amplitudes for Higgs plus jet production involving a modified trilinear Higgs coupling, JHEP 04 (2019) 062 [arXiv:1902.05480] [INSPIRE]. 
[78] H.H. Patel, Package-X: A Mathematica package for the analytic calculation of one-loop integrals, Comput. Phys. Commun. 197 (2015) 276 [arXiv:1503.01469] [INSPIRE].

[79] E. Braaten, C.-S. Li and T.-C. Yuan, The Evolution of Weinberg's Gluonic CP Violation Operator, Phys. Rev. Lett. 64 (1990) 1709 [INSPIRE].

[80] E. Braaten, C.S. Li and T.C. Yuan, The Gluon Color-Electric Dipole Moment and Its Anomalous Dimension, Phys. Rev. D 42 (1990) 276 [inSPIRE].

[81] G. Degrassi, E. Franco, S. Marchetti and L. Silvestrini, QCD corrections to the electric dipole moment of the neutron in the MSSM, JHEP 11 (2005) 044 [hep-ph/0510137] [INSPIRE].

[82] J. de Vries, G. Falcioni, F. Herzog and B. Ruijl, Two- and three-loop anomalous dimensions of Weinberg's dimension-six CP-odd gluonic operator, arXiv:1907.04923 [INSPIRE].

[83] M. Misiak and M. Münz, Two loop mixing of dimension five flavor changing operators, Phys. Lett. B 344 (1995) 308 [hep-ph/9409454] [INSPIRE].

[84] J.A. Gracey, Three loop MS-bar tensor current anomalous dimension in QCD, Phys. Lett. B 488 (2000) 175 [hep-ph/0007171] [INSPIRE].

[85] M. Gorbahn, U. Haisch and M. Misiak, Three-loop mixing of dipole operators, Phys. Rev. Lett. 95 (2005) 102004 [hep-ph/0504194] [INSPIRE].

[86] T. Bhattacharya, V. Cirigliano, R. Gupta, H.-W. Lin and B. Yoon, Neutron Electric Dipole Moment and Tensor Charges from Lattice QCD, Phys. Rev. Lett. 115 (2015) 212002 [arXiv: 1506. 04196] [INSPIRE].

[87] PNDME collaboration, Iso-vector and Iso-scalar Tensor Charges of the Nucleon from Lattice QCD, Phys. Rev. D 92 (2015) 094511 [arXiv:1506.06411] [InSPIRE].

[88] R. Gupta, B. Yoon, T. Bhattacharya, V. Cirigliano, Y.-C. Jang and H.-W. Lin, Flavor diagonal tensor charges of the nucleon from $(2+1+1)$-flavor lattice QCD, Phys. Rev. D 98 (2018) 091501 [arXiv: 1808.07597] [INSPIRE].

[89] M. Pospelov and A. Ritz, Neutron EDM from electric and chromoelectric dipole moments of quarks, Phys. Rev. D 63 (2001) 073015 [hep-ph/0010037] [INSPIRE].

[90] O. Lebedev, K.A. Olive, M. Pospelov and A. Ritz, Probing CP-violation with the deuteron electric dipole moment, Phys. Rev. D 70 (2004) 016003 [hep-ph/0402023] [INSPIRE].

[91] J. Hisano, J.Y. Lee, N. Nagata and Y. Shimizu, Reevaluation of Neutron Electric Dipole Moment with QCD Sum Rules, Phys. Rev. D 85 (2012) 114044 [arXiv:1204.2653] [INSPIRE].

[92] D.A. Demir, M. Pospelov and A. Ritz, Hadronic EDMs, the Weinberg operator and light gluinos, Phys. Rev. D 67 (2003) 015007 [hep-ph/0208257] [INSPIRE].

[93] U. Haisch and A. Hala, Sum rules for CP-violating operators of Weinberg type, arXiv: 1909.08955 [INSPIRE].

[94] I.I.Y. Bigi and N.G. Uraltsev, Induced Multi-Gluon Couplings and the Neutron Electric Dipole Moment, Nucl. Phys. B 353 (1991) 321 [inSPIRE].

[95] Particle Data Group, Review of Particle Physics, Phys. Rev. D 98 (2018) 030001 [INSPIRE].

[96] P. Schmidt-Wellenburg, The quest to find an electric dipole moment of the neutron, arXiv:1607.06609 [INSPIRE]. 
[97] T.M. Ito et al., Performance of the upgraded ultracold neutron source at Los Alamos National Laboratory and its implication for a possible neutron electric dipole moment experiment, Phys. Rev. C 97 (2018) 012501 [arXiv:1710.05182] [InSPIRE].

[98] T. Bhattacharya, V. Cirigliano, R. Gupta, E. Mereghetti and B. Yoon, Dimension-5 CP-odd operators: QCD mixing and renormalization, Phys. Rev. D 92 (2015) 114026 [arXiv: 1502.07325] [INSPIRE].

[99] T. Bhattacharya, V. Cirigliano, R. Gupta and B. Yoon, Quark Chromoelectric Dipole Moment Contribution to the Neutron Electric Dipole Moment, PoS (LATTICE2016) 225 (2016) [arXiv: 1612.08438] [INSPIRE].

[100] M. Abramczyk, S. Aoki, T. Blum, T. Izubuchi, H. Ohki and S. Syritsyn, Lattice calculation of electric dipole moments and form factors of the nucleon, Phys. Rev. D 96 (2017) 014501 [arXiv: 1701.07792] [INSPIRE].

[101] J. Dragos, T. Luu, A. Shindler and J. de Vries, Electric Dipole Moment Results from lattice QCD, EPJ Web Conf. 175 (2018) 06018 [arXiv:1711.04730] [INSPIRE].

[102] M. Rizik, C. Monahan and A. Shindler, Renormalization of CP-Violating Pure Gauge Operators in Perturbative QCD Using the Gradient Flow, PoS (LATTICE2018) 215 (2018) [arXiv: 1810.05637] [INSPIRE].

[103] J. Kim, J. Dragos, A. Shindler, T. Luu and J. de Vries, Towards a determination of the nucleon EDM from the quark chromo-EDM operator with the gradient flow, PoS (LATTICE2018) 260 (2019) [arXiv: 1810.10301] [INSPIRE].

[104] T. Bhattacharya, B. Yoon, R. Gupta and V. Cirigliano, Neutron Electric Dipole Moment from Beyond the Standard Model, arXiv:1812.06233 [INSPIRE].

[105] S. Syritsyn, T. Izubuchi and H. Ohki, Calculation of Nucleon Electric Dipole Moments Induced by Quark Chromo-Electric Dipole Moments and the QCD $\theta$-term, PoS (Confinement2018) 194 (2019) [arXiv: 1901.05455] [INSPIRE].

[106] R. Gupta, Present and future prospects for lattice QCD calculations of matrix elements for nEDM, PoS (SPIN2018) 095 (2019) [arXiv: 1904.00323] [INSPIRE].

[107] USQCD collaboration, The Role of Lattice QCD in Searches for Violations of Fundamental Symmetries and Signals for New Physics, arXiv:1904.09704 [INSPIRE].

[108] T. Hahn, Generating Feynman diagrams and amplitudes with FeynArts 3, Comput. Phys. Commun. 140 (2001) 418 [hep-ph/0012260] [INSPIRE].

[109] A. Alloul, N.D. Christensen, C. Degrande, C. Duhr and B. Fuks, FeynRules $2.0-A$ complete toolbox for tree-level phenomenology, Comput. Phys. Commun. 185 (2014) 2250 [arXiv:1310.1921] [INSPIRE]. 